\title{
DYNAMICS OF INTERACTING CLUSTERS AND DIELECTRIC RESPONSE IN RELAXOR FERROELECTRICS
}

\author{
B.E.Vugmeister and H. Rabitz \\ Department of Chemistry, Princeton University, Princeton, NJ 08544
}

\begin{abstract}
The dielectric response in relaxor ferroelectrics is analyzed in the framework a model for the polarization dynamics in the presence of polar clusters. We associate the origin of polar clusters with the atoms displaced from their centrosymmerical positions even above $T_{c}$. Their collective hopping in multi-well potentials induced by disorder is analogous with the situation in glasses. The theory explicitly takes into account the distribution of cluster reorientation frequencies and the effect of cluster-cluster interactions in highly polarizable crystals, which we describe in terms of the local field distribution function. The dielectric constant is obtained from an integral master equation for the polarization dynamics in the presence of a time dependent electric field. The theory is applied for the analysis of the shape of the frequency dependent permittivity in the typical relaxor ferrolectrics $P S T$ as a function of temperature. The comparison of the theory with experiment shows that in contrast to earlier assumptions, the observed Vogel-Fulcher dependence of the permittivity maximum is a consequence of the Vogel-Fulcher temperature dependence of the cluster reorientation frequency.
\end{abstract}

PACS numbers: 77.80.-e, 64.60.-i

Typeset using REVTEX 


\section{INTRODUCTION}

In the family of ferroelectrics a large group of mixed and disordered "relaxors" has been identified including perovskites $\mathrm{PbMg}_{1 / 3} \mathrm{Nb}_{2 / 3} \mathrm{O}_{3}(\mathrm{PMN}), \mathrm{PbSc}_{1 / 2} \mathrm{Ta}_{1 / 2} \mathrm{O}_{3}$ (PST), Lamodified $\mathrm{PbZr}_{1-x} \mathrm{Ti}_{x} \mathrm{O}_{3}$ (PLZT), tungsten-bronze structure oxides like $\mathrm{Pb}_{x} \mathrm{Ba}_{1-x} \mathrm{Nb}_{2} \mathrm{O}_{6}$ (PBN), etc. [1-15] Due to the effect of configurational disorder the properties of relaxor ferroelectrics are very different from those in translationally invariant ferroelectrics. Among the unusual properties of these materials are a) the coexistence of slow kinetics typical for spin glasses with a very large dielectric constant indicating intermideate range polar order on the nanometer length scale which can be transformed to true long range order, (i.e., to macroscopic polarization and strain) by a suitable change of the composition or by applying an external electric field; b) the existence of a frequency dependent slim hysteresis loop even above the transition temperature; c) the difference between field-cooled and zero fieldcooled dielectric susceptibility, etc. All these findings indicate that the observed properties of relaxor ferroelectrics are nonequilibrium properties. Relaxor ferroelectrics thus represent a new low temperature state of polar dielectrics compared with conventional ferroelectrics.

Experiments show that there is symmetry breaking on a nanometer scale as observed from Raman scattering [3] and X-ray and neutron diffraction [5,9]. This observation implies that polar clusters exist even well above $T_{c}$ and the observed properties of relaxors are strongly affected by the reorientations of the clusters. In particular, cluster reorientation in the applied electric field induces strong polar-strain coupling which makes relaxor ferroelectrics the candidate materials for the next generation of ultrasonic transducers 16].

It has been proposed [2], in analogy with the cluster model of spin glasses, that the polar clusters behave like large superparaelectric dipole moments. The broad distribution of relaxation times for cluster orientations originates from the distribution of the potential barriers separating different orientational states. The superparaelectric model [2] based on the assumption of independent clusters can not explain, however, the appearance of long range order with the change of the material composition or due to an applied external field. 
The appearance of long range order also can not be explained by the inclusion of frustrated interactions between superparaelectric moments [4] that lead to the dipole spin glass state. An alternative proposition [6,13] [15] relates the origin of relaxor behavior to the domain states induced by the static random field caused, for example, by charged composition fluctuations. Although this model seems very attractive, it encounters difficulties in explaining the observed cluster dynamics in the high temperature phase.

We will show that the proposed model is capable to describe the anomalies of dielectric response of relaxor ferroelectrics and can be applied for systems possessing first or second order phase transitions or remaining only incipient ferroelectrics with very high dielectric constant and vanishing spontaneous polarization.

The origin of polar clusters in relaxor ferroelectrics is still not understood. It has been proposed [10] that off-center ions might be responsible for relaxor properties in analogy with $K_{1-x} \mathrm{Li}_{x} \mathrm{TaO}_{3}$ (KLT) where $\mathrm{Li}$ impurity ions occupy the off-center positions near the vacant $K$ sites. It is well established [17] that in the case of impurity induced relaxors like KLT the single impurity potential possesses multi-well structure which allows the thermal jumps of off-center ions between different potential minima. In contrast, in disordered complex perovskites the displacement of atoms from their centro-symmetrical positions is caused apparently by the charge compositional fluctuations, which violate the charge balance within the adjacent unit cells and lead to the additional electrostatic forces on the atoms. In this situation one would not expect that the single atom potential energy possesses multiminima structure. Atom reorientations would more probably take place if they associate with the collective motion of atoms within small clusters, (i.e., the cluster potential energy is characterized by the multi-well (double well) structure).

Such a picture is analogous to the situation in glasses where the origin of two level systems responsible for glass anomalies is associated with the double well potentials [19, [8]. It is generally assumed that the existence of the double well potentials is due to the disorder in glasses, so that local rearrangements of atoms might switch the system between two adjacent local energy minima. Attempts to detect double well potentials in computer simulations [20] 
based on the assumption of the motion of single atoms 21] have been unsuccessful. Instead the origin of double well potentials in glasses has been successfully explained [22] in terms of collective motion of atoms within small clusters.

In this paper we develop further the cluster model of relaxor ferroelectrics taking explicitly into account a) the broad distribution of local field experienced by each cluster due to cluster-cluster interaction and b) the broad distribution of potential barriers controlling cluster dynamics. We will show that the proposed model is capable of describing the anomalies of dielectric response of relaxor ferroelectrics and can be applied for systems possessing first or second order phase transitions or remaining an incipient ferroelectrics with very high dielectric constant and vanishing spontaneous polarization.

\section{MODEL}

Based on the analogy between disorder induced double well potentials in glasses and disordered relaxor ferroelectrics we will adopt the following picture of the cluster dynamics in relaxor ferroelectrics. Each minimum in a double well potential for a given cluster is characterized by the cluster dipole moment or cluster polarization $P_{c l}$. The potential barrier between different minima determines the atom hopping frequency $\tau^{-1}$. Clusters interact with each other, and interacting reorientable polarizable clusters should increase the crystal dielectric response. This conclusion is supported by the experiment [7] in PST where the effect of disorder in the relative occupation of B sites by Sc or Ta atoms dramatically increases the dielectric constant.

Time or frequency dependent dielectric response can be analyzed with the use of the master equation

$$
\frac{d P_{c l}}{d t}=-\frac{1}{\tau}\left(P_{c l}-P_{c l}^{e q}\left(E_{L}\right)\right)
$$

well known from the theory of ferroelectrics of order-disorder type 23,24, describing the

relaxation of the polarization of each cluster to its quasi-equilibrium value $P_{c l}^{e q}\left(E_{L}\right)$ which 
depends on the value of the local field $E_{L}$ induced by other clusters at any moment of time. In general the local field $E_{L}$ is a time dependent random field. It also includes the contribution from the applied field $E_{e x}$ and the contribution from the static random fields caused by the material imperfections.

In order to apply Eq.(II) to relaxor ferroelectrics one should perform the average over the distribution of relaxation times $\tau$ and the distribution of the local fields $E_{L}$. For this purpose we rewrite Eq.(11) in the equivalent integral form and take the average with respect to $\tau, E_{L}$ and the initial cluster polarization $P_{c l}(0)$ to obtain

$$
P(t)=P(0) \tilde{Q}(t)-\int_{0}^{t} d t^{\prime} \frac{\partial \tilde{Q}\left(t-t^{\prime}\right)}{\partial t} P^{e q}\left(t^{\prime}\right) .
$$

The function $\tilde{Q}(t)=\overline{e^{-t / \tau}}$, where the overbar denotes the average over the relaxation time $\tau$, characterizes the slow nonexponential kinetics of the system. In addition

$$
P^{e q}(t)=\int d E P_{c l}^{e q}(E) f(E, P(t))
$$

where $f(E, P(t))$ is the distribution function of the local field which depends parametrically on the value of the average polarization of the system $P(t)$. We will consider below $f(E, P)$ in the form $f(E, P)=\tilde{f}\left(E-\gamma P-\gamma \epsilon_{0} E_{e x} / 4 \pi\right)$. This form of $f$ is consistent with the mean field approximation $f(E, P)=\delta\left(E-\gamma P-\gamma \epsilon_{0} E_{e x} / 4 \pi\right)$, where $\delta$ is the delta-function and $\gamma$ is the local field phenomenological parameter. In disordered systems the effect of composition fluctuations leads to a deviation from the simple mean field picture that can be taken into account by the replacement of the $\delta$-function by the function $\tilde{f}$ with finite width. The shape and the width of $\tilde{f}(E)$ depend on the explicit form of the cluster-cluster interactions as well as on static random fields caused by the material imperfections.

The value $\gamma \epsilon_{0} E_{e x} / 4 \pi$ is the local field induced by the external field in the dielectric media with the dielectric constant $\epsilon_{0}>>1$ [25] (i.e., we assume that the polar clusters are distributed in a highly polarizable dielectric media). In relaxor ferroelectrics which are mainly perovskite-based highly polarizable materials, the typical values are $\epsilon_{0}>10^{3}$.

Eq.(2) can be applied for the analysis of different experimental situations (e.g., decay 
of the polarization, the difference between field-cooled and zero field-cooled dielectric susceptibility, the effect of a frequency dependent hysteresis loop, etc). In this paper we will concentrate, for illustration, on the calculation of the frequency dependent linear dielectric susceptibility. In order to calculate the steady state susceptibility in the presence of an additional time independent field $E_{e x}^{(0)}$ we write $E_{e x}(t)=E_{e x}^{(0)}+E_{e x}^{(1)} e^{i \omega t}$ and $P(t)=P_{s}+P_{1}(t)$, where $P_{s}$ is the time independent polarization induced by the field $E_{e x}^{0}$ (for $E_{e x}^{(0)}=0$, we denote $P_{s}$ as the spontaneous polarization). We obtain from Eq.(2) the following self consistent equation for $P_{s}$

$$
P_{s}=\int d E P_{c l}^{e q}(E) f\left(E, P_{s}\right)
$$

Assuming $P(0)=P_{s}$ in Eq.(2), considering a linear expansion of $P^{e q}(t)$ with respect to $E_{e x}^{(1)}$ and $P_{1}(t)$, and taking Laplace transform of Eq.(2), we obtain

$$
\epsilon(\omega, T)=\frac{\epsilon_{0}}{1-\kappa(T) Q(\omega, T)}
$$

where we used the definition of the dielectric constant $\epsilon(\omega, T)=4 \pi \partial P_{1}(\omega) / \partial E_{e x}^{(1)}+\epsilon_{0}$. In Eq.(5)

$$
\kappa(T)=\int d E P_{c l}^{e q}(E) \frac{\partial f\left(E, P_{s}\right)}{\partial P_{s}}
$$

and $Q(\omega, T)$ is the Laplace transform of $\partial \tilde{Q(t)} / \partial t$ given by

$$
Q(\omega, T)=\overline{(1 /(1+i \omega \tau))}
$$

Assuming an Arrhenius or Vogel-Fulcher (VF) law for $\tau$

$$
\tau(T)=\tau_{0} \exp \left[U /\left(T-T_{0}\right)\right]
$$

we may calculate $Q(\omega, T)$. Thus, we obtain for $Q^{\prime}(\omega, T)$ 26

$$
Q^{\prime}(\omega, T)=\int_{0}^{\left(T-T_{0}\right) \ln \frac{1}{\omega \tau_{0}}} d U g(U)
$$

where $g(U)$ is the distribution function of the potential barriers. 
In the spirit of Landau phenomenological theory one can further expand the right hand side of Eqs. (44) and (6) in a power series with respect to $P_{s}$ assuming that the applied field is sufficiently small, and present $\kappa$ as

$$
\kappa=a_{1}+3 a_{3} P_{s}^{2}+5 a_{5} P_{s}^{4}
$$

Eqs.(5), (9), and (10) give the phenomenological description of the dynamical response of relaxor ferroelectrics and can be applied for systems possessing first or second order phase transitions or remaining as only incipient ferroelectrics with very high dielectric constant and vanishing spontaneous polarization.

\section{AMOMALIES IN THE DIELECTRIC RESPONSE}

In this section we illustrate a capability of the model in the description of the dielectric response of relaxor ferroelectrics.

Relaxors with incipient ferroelectric order. In order to simultaneously reproduce the high values of the dielectric constant in relaxor ferroelectrics and the absence of spontaneous polarization (like that in PMN or PST with vacancies) one should assume that $a_{3}<0$ and $a_{1}(T) \rightarrow 1$ remaining, however, less than 1 at all temperatures. We chose for the illustrative calculations $a_{1}(T)=0.95 \tanh (0.5 / T)$ (which, according to Eq.(5)), reproduces Curie-like high temperature behavior of $\epsilon(0, T)$ and its high saturation value at low temperatures) and $g(U)=2.5 U^{4} /\left(0.5+U^{5}\right)^{2}$. We assume also the Arrhenius temperature dependence of $\tau(T)$, i.e. $T_{0}=0$ in Eq.(8). Using the chosen values of $a_{1}(T), g(U)$, and $T_{0}$ we calculated the dielectric permittivity $\epsilon$ as a function of temperature for different values of $\ln \left(\frac{1}{\omega \tau_{0}}\right)$.

The calculated real part of permittivity is shown in Fig.1 Note that the frequency dependent maximum of $\epsilon^{\prime}$ is not just a relaxation maximum. It originates from the competitive temperature dependences of $Q^{\prime}(T)$ and $a_{1}(T)$. The behavior obtained for $\epsilon^{\prime}$ is in reasonable qualitative agreement with the experiment [6,0,10]. For a more detailed comparison with the experiment one needs to find the functions $Q(\omega, T)$ and $a_{1}(T)$ corresponding to the best fit with the experimental data. 
Manifestation of the first order phase transition. In some relaxor ferroelectrics (e.g., disordered PST or KLT above the critical concentration of Li ions) there is evidence of a first order phase transition, which manifests itself in the sharp drop of the dielectric constant at the temperature below the position of the relaxation maximum. In order to reproduce this behavior within the proposed phenomenological theory one should assume $a_{3}>0$. For example for $a_{3}=1.7, a_{5}=-11$ and the values of $a_{1}(T)$ and $Q^{\prime}$ being the same as used above, the first order phase transition occurs at $T_{c} \approx 0.2$ (in the chosen dimensionless units).

The calculated values of $\epsilon^{\prime}$ are shown in Fig.2. A remarkable feature of the $\epsilon^{\prime}$ temperature dependence is that the relaxation maximum of $\epsilon^{\prime}$ approaches the phase transition temperature with a decrease of the frequency until it finally disappears transforming to a sharp peak like that in conventional ferroelectrics. This behavior is in qualitative agreement with that observed in PST.

Reconstruction of the relaxation function $Q(\omega, T)$. PST with B-site chemical disorder undergoes a first order relaxor-ferroelectric phase transition at $T_{c} \approx 269 \mathrm{~K}$ [7]. The dielectric permittivity of PST shows pronounced frequency dispersion with the position of a frequency dependent maximum obeying the VF law [7] $\omega=\omega_{0} \exp \left[-U /\left(T_{m}-T_{0}\right)\right]$, where $\omega$ is the frequency of the applied field and $T_{m}$ is the temperature of the permittivity maximum corresponding to the frequency $\omega$. It has been widely accepted (see, e.g., [27]) that the VF type relation for the permittivity maximum is a consequence of the VF law (8) for $\tau(T)$. This assumption has been argued recently by Tagantsev [28] who proposed that the observation in PST of the VF frequency dependence of $T_{m}$ can be explained with the use of the Arrhenius temperature dependence of $\tau$ taking into account the existence of the first order phase transition and the fact that in PST $T_{0} \approx T_{c}$. An important conclusion of Tagantsev's analysis is the indication that $T_{m}$ might be influenced significantly by the values of static permittivity, not only by the specific temperature dependence of $\tau$. However, in order to be more conclusive one should analyze the shape of the frequency dependent permittivity as a function of temperature for different frequencies, not just the frequency dependence of the permittivity maximum. Such an analysis is given below with the use of Eq.(5). 
First, we extrapolated the experimental data [7] and extracted the static permittivity above $T_{c}$ as shown in Fig.3 (dotted curve). Then, using Eq.(5) and the fact that at $\omega=0 Q^{\prime}$ is equal to 1 , we obtained the values of $\kappa(T)$ above $T_{c}$. We used the value $\epsilon_{0} \approx 500$ for the host lattice permittivity based on the experimental low temperature values of $\epsilon(T)$ where the polar clusters are frozen. With the values obtained for $\kappa(T)$ and the experimental values of the frequency dependent permittivity $\epsilon^{\prime}\left(\omega_{1}, T\right)$ at frequency $\omega_{1}=10 \mathrm{KHz}$ (curve 1) we have calculated the values of $Q^{\prime}\left(\omega_{1}, T\right)$ using Eq.(5). According to Eq.(9) $Q^{\prime}(\omega, T)$ is a function of $\left(T-T_{0}\right) \ln \left(\omega \tau_{0}\right)$ that results in the scaling relation $Q^{\prime}(\omega, T)=Q^{\prime}\left(\omega_{1}, T_{1}\right)$ with $T_{1}=\left(T-T_{0}\right) \frac{\ln \left(\omega \tau_{0}\right)}{\ln \left(\omega_{1} \tau_{0}\right)}+T_{0}$. Using this relation one can reconstruct the values of $Q^{\prime}(\omega, T)$ at other frequencies and, therefore, the values of $\epsilon^{\prime}(\omega, T)$. The results of such a reconstruction are presented in Fig.3 for the upper $100 \mathrm{~Hz}$ (curve 2) and lower $1 \mathrm{MHz}$ (curve 3) boundary frequencies used in the experiment. We used $\tau_{0} \approx 10^{-12}$ in accordance with Ref. [0].

One can see from Fig.3 that the approach reproduces rather well the shape of the temperature dependence of the permittivity at different frequencies of the applied field. The parameter of the fit $T_{0}$ is found to be $T_{0} \approx 258 \mathrm{~K}$ which is in reasonable agreement with the value $T_{0} \approx 265 K$ estimated in Ref. [7]. This result means that the employment of the VF law for $\tau(T)$ is very crucial for obtaining the correct temperature and frequency dependence of the permittivity for PST. The VF relation for $\tau(T)$ results simultaneously in the very fast temperature dependence of the permittivity and its rather slow dependence on $\ln \left(\omega \tau_{0}\right)$, observed in the experiment. At the same time an Arrhenius like dependence of $\tau(T)$ would result in an extremely rapid dependence of the permittivity on $\ln \left(\omega \tau_{0}\right)$, which is inconsistent with the experimental data.

We are grateful to A. Khachaturian, A. Tagantsev, and J. Toulouse for the useful discussions. This work is supported by the National Science Foundation and the Army Research Office. 


\section{REFERENCES}

[1] G.A. Smolensky et al., Ferroelectrics and Related Materials (Gordon and Breach, New York, 1984)

[2] L.E. Cross, Ferroelectrics 76,241 (1987).

[3] G. Burns and F.H. Dagol, Ferroelectrics 104, 25 (1990).

[4] D. Vieland, S.J. Jang, L.E. Cross, and M. Wuttig, J.Appl. Phys. 68, 2916 (1990).

[5] N. de Mathan, E. Husson, G. Galvarin, J.R. Gavarri, A.W. Hewat,and A. Morell, J. Phys. Condens. Matter 3, 8159 (1991).

[6] V. Westphal, W. Kleemann, and M.D. Glinchuk, Phys. Rev. Lett. 68,847 (1992).

[7] F. Chu, N.Setter, and A.K.Tagantsev, J.Appl.Phys.74, 129 (1993).

[8] R. Sommer, N.K.Yushin, and J.J. van der Klink, Phys. Rev. B 48, 13230 (1993).

[9] H.D. Rosenfeld, and T.Egami, Ferroelectrics 158, 351 (1994).

[10] J.Toulouse, B.E.Vugmeister, and R. Patnaik, Phys. Rev. Lett. 73,3467 (1994).

[11] E.V. Cola, E.Yu. Koroleva, N.N. Okuneva, and S.B. Vakhrushev. Phys. Rev. Lett. 74, 1681,1994 .

[12] G. Samara, Phys.Rev.Lett. 77, 314 (1996).

[13] A.E. Glazounov, A.K. Tagantsev, and A.J. Bell, Phys. Rev. B 53,11281 (1996).

[14] H. Qian and L.A. Bursill, Int. J. Mod. Phys. B 10, 2027 (1996).

[15] N. Semenovskaya and A. Khachaturian, Ferroelectrics (1997) (in press).

[16] S.-E. Park and T.R. Shrout, J. Mat. Res. Innov. (1997) (in press).

[17] B.E. Vugmeister and M.D.Glinchuk, Rev.Mod.Phys. 62, 991 (1990).

[18] W. A. Phillips, J. Low Temp. Phys. 7, 351 (1972). 
[19] P.W. Anderson, B.I. Halperin and C. M. Varma, Philos. Mag. 25, 1, (1972).

[20] T. A. Weber and F. H. Stillinger, Phys. Rev. B 32, 5402 (1985).

[21] R. Harris and L.J. Lewis, Phys. Rev. B 25, 4997 (1982).

[22] A. Heuer and R.J. Silbey, Phys. Rev. Lett. 70, 3911 (1993); Phys. Rev. B 53, 609 (1996).

[23] R.Blinc and B.Zeks, Soft Modes in Ferroelectrics and antiferroelectrics (North-Holland, New York, 1974).

[24] M.E. Lines and A.M. Glass, Principles and Applications of Ferroelectrics (Clarendon Press, Oxford, 1977).

[25] C.Kittel, Introduction to Solid State Physics (Willey, New York, 1986).

[26] E.Courtens, Phys.Rev.Lett, 52, 69 (1984).

[27] K. Binder and A.P. Young, Rev.Mod.Phys. 58, 801 (1986).

[28] A.Tagantsev, Phys.Rev.Lett. 72, 1100 (1994). 


\section{FIGURES}

FIG. 1. Real part of the dielectric susceptibility in relaxors with incipient ferroelectric order.

FIG. 2. Effect of the first order phase transition on the dielectric response in relaxor ferroelectrics.

FIG. 3. $\epsilon^{\prime}(\mathrm{T})$ for disordered PST above $T_{c}$ for different frequencies. The vertical line shows the phase transition temperature. Solid lines 2,3 and the circles are respectively the reconstructed and the experimental values of $\epsilon^{\prime}(T)$. The error bars indicate the uncertainties of the reconstruc-

tion due to the uncertainties in the values obtained for $\kappa(T)$ by the extrapolation(dotted line) of experimental data. 


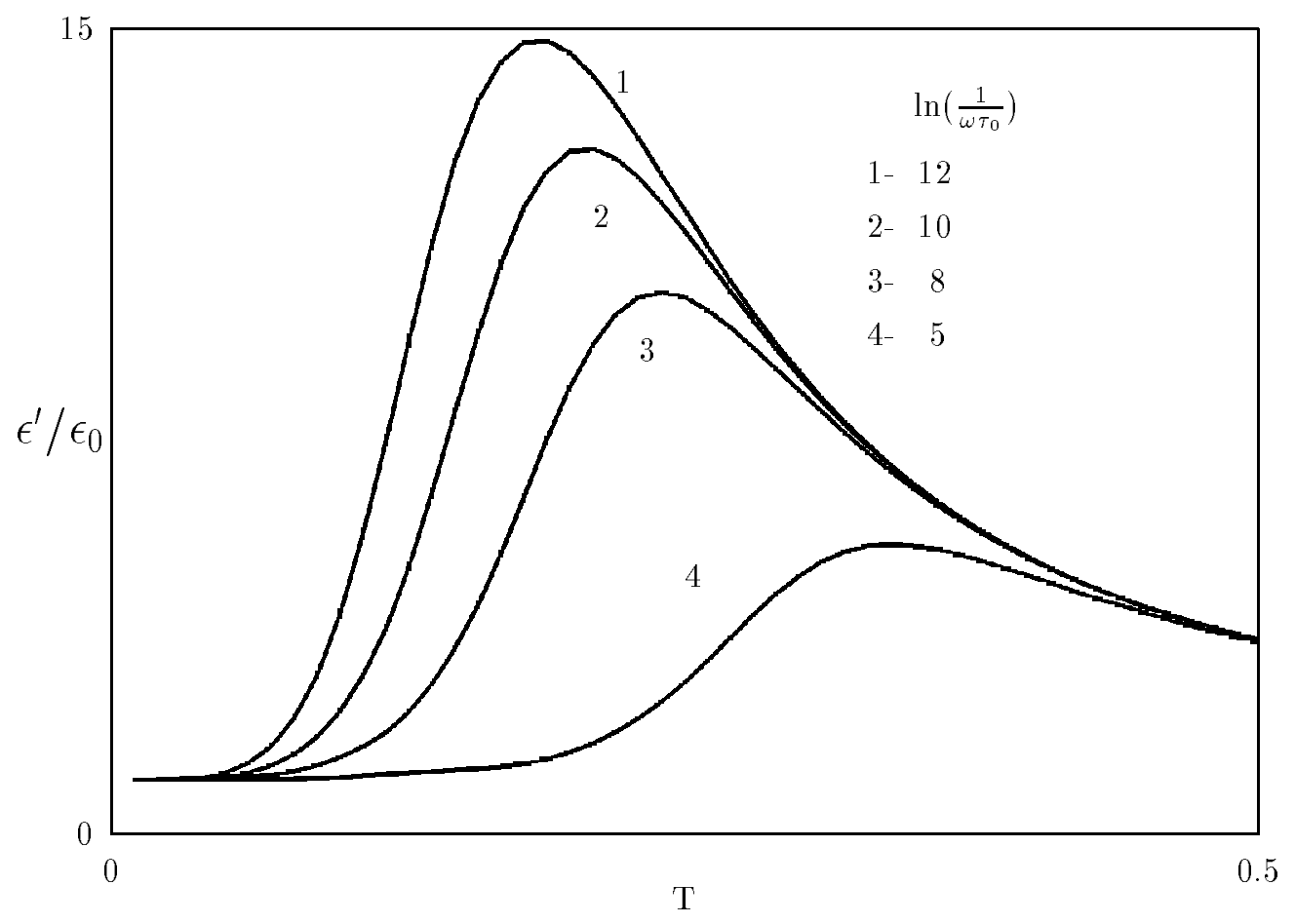

Fig.1 


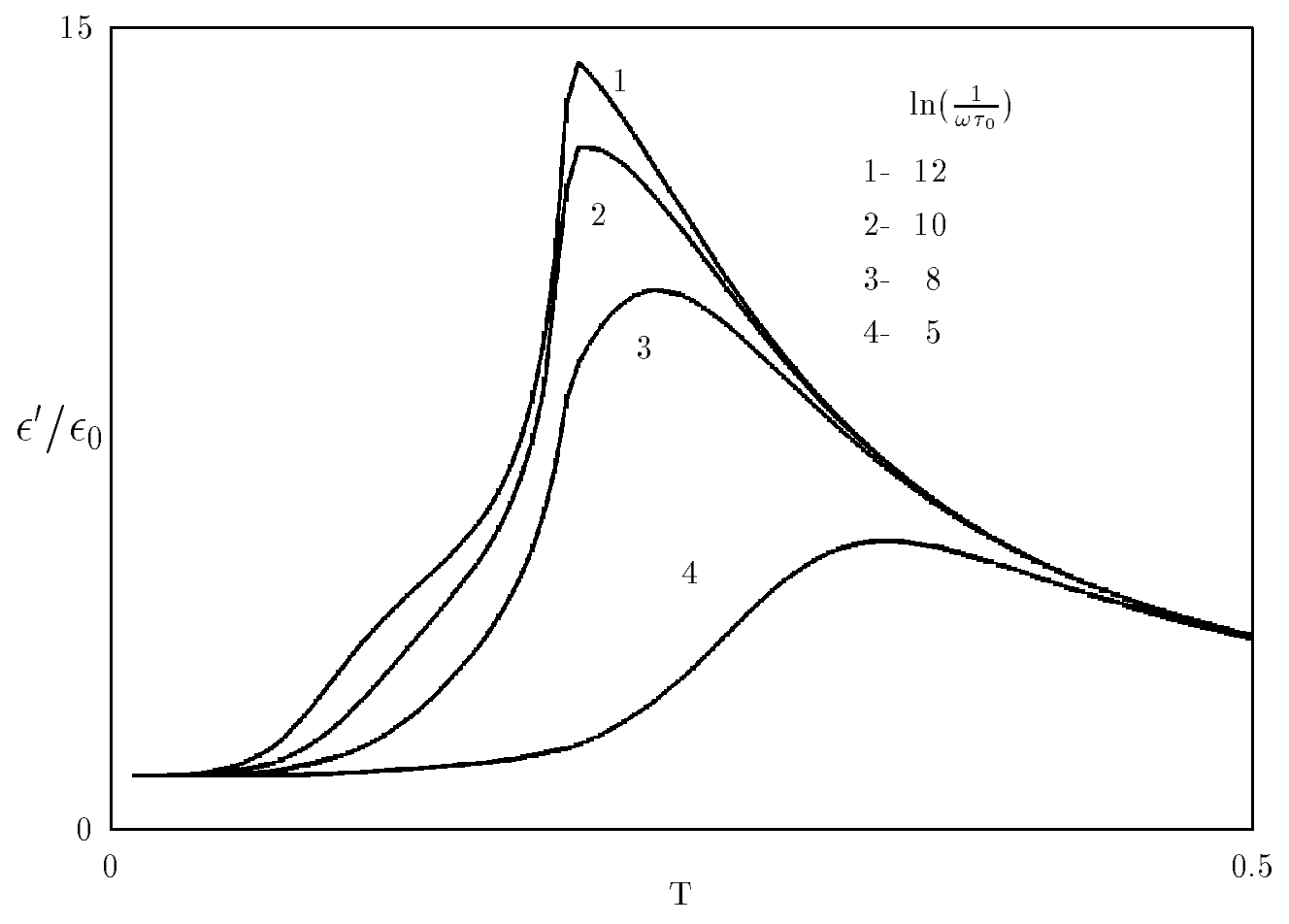

Fig.2 


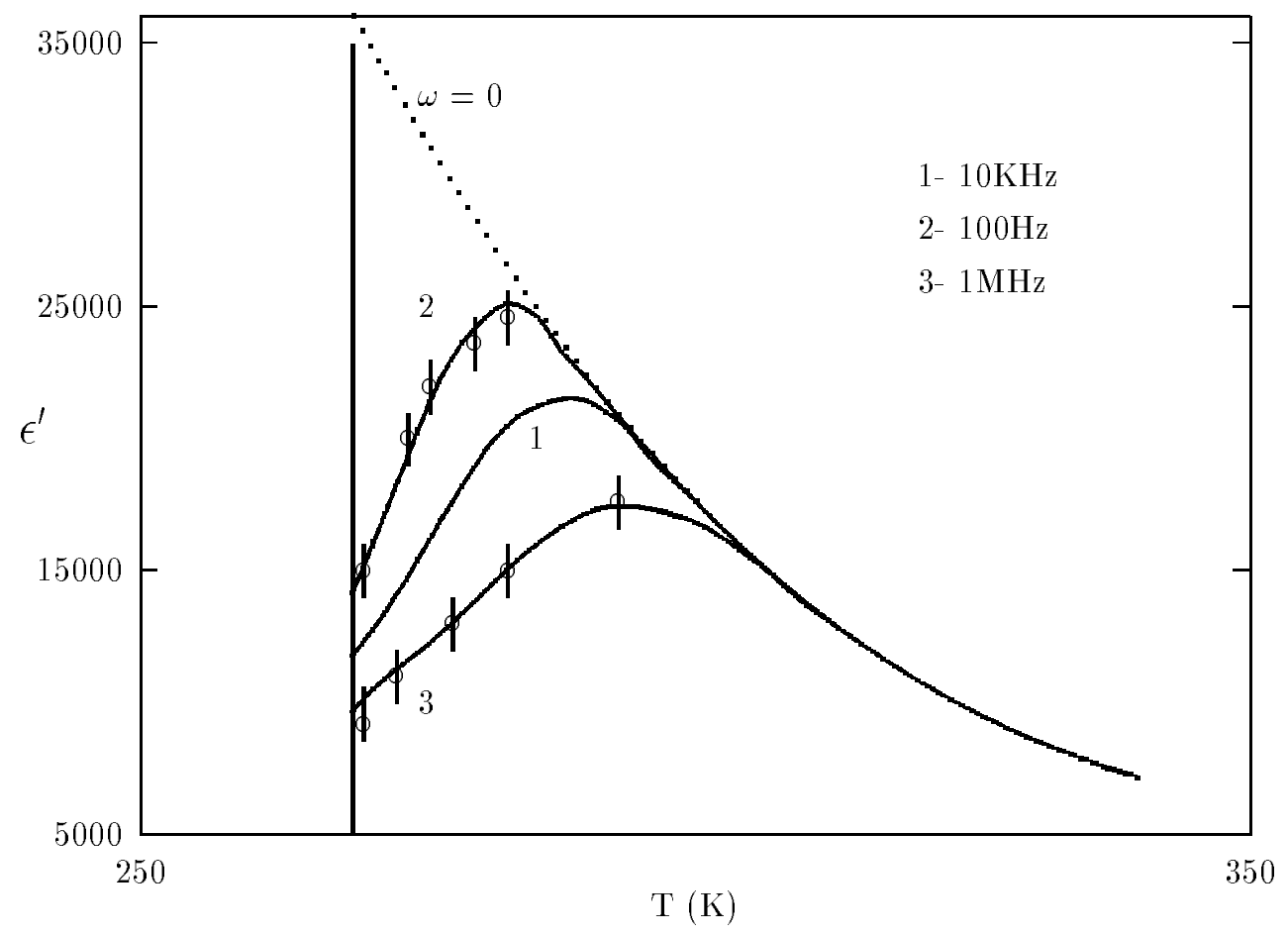

Fig.3 